\title{
Comparação entre Nifedipina por Via Sublingual e Clonidina por Via Venosa no Controle de Hipertensão Arterial Peri-Operatória em Cirurgias de Catarata *
}

\section{Comparison between Sublingual Nifedipine and Intravenous Clonidine to Control Perioperative Arterial Hypertension in Cataract Procedures}

Renato Mestriner Stocche, TSA ${ }^{1}$, Luis Vicente Garcia, TSA ${ }^{1}$, Jyrson Guilherme Klamt, TSA ${ }^{1}$, Alexandre Pachione, $T_{S A}{ }^{2}$, Huang $\mathrm{HYu}^{3}$, Waleska A Oliveira ${ }^{3}$

\section{RESUMO}

Stocche RM, Garcia LV, Klamt JG, Pachione A, Yu HH, Oliveira WA - Comparação entre Nifedipina por Via Sublingual e Clonidina por Via Venosa no Controle de Hipertensão Arterial PeriOperatória em Cirurgias de Catarata

\begin{abstract}
Justificativa e Objetivos - Estudo recente mostra que a clonidina por via venosa apresenta-se eficaz e segura no tratamento de crises hipertensivas durante cirurgia de catarata. Este estudo visa comparar o uso de nifedipina, droga amplamente utilizada por via sublingual, e clonidina por via venosa no controle da hipertensão arterial em cirurgias de catarata.

Método - Setenta e cinco pacientes submetidos à facectomia foram distribuídos de forma aleatória e encoberta em: Grupo A, que recebeu nifedipina e Grupos C2 e C3, que receberam, respectivamente, 2 e $3 \mu \mathrm{g} . \mathrm{kg}^{-1}$ de clonidina por via venosa. Todos os pacientes apresentavam hipertensão arterial (PAS > $170 \mathrm{mmHg}$ ou $P A D>110 \mathrm{mmHg}$ ). As $P A S, P A D$ e freqüência cardíaca (FC) foram medidas e comparadas nos momentos: 0 (antes do tratamento) e de 2 em 2 minutos até o final do procedimento. Os eventos adversos foram anotados.
\end{abstract}

Resultados - Após o tratamento ocorreram diminuições da PAS e PAD nos 3 grupos $(p<0,001)$. Houve controle da pressão arterial ( $<160 \mathrm{mmHg}$ ) em $32 \%, 64 \%$ e $72 \%$ dos pacientes nos grupos $A, C 2$ e $C 3$, respectivamente $(p<0,05)$. No grupo $C 3$ ocorreu maior incidência de efeitos colaterais que nos grupos C2 e $A(p<0,05)$.

Conclusões - A clonidina por via venosa é mais eficaz que a nifedipina, por via sublingual, no controle de crises hipertensivas no peri-operatório de cirurgias de catarata. Contudo, a dose de $3 \mu \mathrm{g} \cdot \mathrm{kg}^{-1}$ pode estar relacionada a efeitos colaterais, devendo-se iniciar o tratamento com $2 \mu \mathrm{g} . \mathrm{kg}^{-1}$.

UNITERMOS: CIRURGIA, Oftalmológica: catarata; COMPLICAÇÕES: hipertensão arterial, hipotensão arterial, bradicardia; DROGAS: clonidina, nifedipina

\footnotetext{
* Recebido do (Received from) Hospital das Clínicas de Ribeirão Preto USP

1. Professor da Faculdade de Medicina de Ribeirão Preto - USP

2. Médico Assistente do HCRP - USP

3. ME do CET/SBA do HCRP-USP
}

Apresentado (Submitted) em 11 de setembro de 2001 Aceito (Accepted) para publicação em 20 de dezembro de 2001

Correspondência para (Mail to):

Dr. Renato Mestriner Stocche

Rua Adolfo Serra, 237 - Alto da Boa Vista

14025-520 Ribeirão Preto, SP

(c) Sociedade Brasileira de Anestesiologia, 2002

\section{SUMMARY}

Stocche RM, Garcia LV, Klamt JG, Pachione A, Yu HH, Oliveira WA - Comparison between Sublingual Nifedipine and Intravenous Clonidine to Control Perioperative Arterial Hypertension in Cataract Procedures

Background and Objectives - A recent study has shown that intravenous clonidine is effective and safe in treating perioperative arterial hypertension during cataract procedures. This study aimed at comparing sublingual nifedipine and intravenous clonidine to control arterial hypertension during cataract procedures.

Methods - Participated in this randomized double-blind study 75 patients submitted to facectomy, who were distributed in: Group $A$, receiving nifedipine and Groups $C 2$ and $C 3$, receiving 2 and $3 \mu \mathrm{g} \cdot \mathrm{kg}^{-1}$ intravenous clonidine, respectively. All patients had arterial hypertension (SBP > $170 \mathrm{mmHg}$ or $D B P>110$ $\mathrm{mmHg}$ ). SBP, DBP and HR were monitored and compared in moments $O$ (before treatment), and at 2-minute intervals until the end of the procedure. Adverse events were recorded.

Results - There has been SBP and DBP decrease in all groups after treatment $(p<0.001)$. There has been blood pressure control $(<160 \mathrm{mmHg}$ ) in $32 \%, 64 \%$ and $72 \%$ of groups $A, C 2$ and $C 3$ patients, respectively $(p<0.05)$. Group C3 presented a higher incidence of side-effects, as compared to groups $C 2$ and $A$ ( $p<$ 0.05).

Conclusions - Intravenous clonidine is more effective than sublingual nifedipine to control perioperative arterial hypertension during cataract extraction. However, the dose of $3 \mu \mathrm{g} . \mathrm{kg}^{-1}$ may be related to side-effects and treatment should be started with $2 \mu \mathrm{g} \cdot \mathrm{kg}^{-1}$.

KEY WORDS - COMPLICATIONS: arterial hypertension, arterial hypotension, bradycardia; DRUGS, clonidine, nifedipine; SURGERY, Ophthalmologic: cataract

\section{INTRODUÇÃO}

$\mathrm{E}_{\mathrm{cos}}^{\mathrm{m}}$ estudo retrospectivo anterior, foi demonstrada a eficácia do uso da clonidina, por via venosa, no controle de crises hipertensivas no período peri-operatório de cirurgia de catarata ${ }^{1}$. Neste estudo foi utilizada a dose fixa de 150 $\mu \mathrm{g}$ não levando em consideração o peso do paciente. Em outro estudo controlado com placebo, foi verificado que a pré-medicação com $150 \mu \mathrm{g}$ de clonidina, por via oral, previne crises hipertensivas sem produzir efeitos adversos em pacientes submetidos à cirurgia de catarata ${ }^{2}$.

Anifedipina por via sublingual tem sido amplamente utilizada no tratamento de crise hipertensiva relacionada ou não a procedimentos cirúrgicos ${ }^{3}$. Contudo, não encontramos na li- 
teratura estudos comparativos entre nifedipina e clonidina no controle pressórico peri-operatório.

O objetivo deste estudo foi comparar a eficácia da nifedipina, por via sublingual, com duas doses de clonidina, por via venosa, no tratamento de crises hipertensivas no período peri-operatório de cirurgia de catarata.

\section{MÉTODO}

Após a aprovação pela Comissão de Ética em Pesquisa do Hospital de Clínicas de Ribeirão Preto e consentimento livre e esclarecido dos pacientes, realizou-se estudo prospectivo, com distribuição aleatória e encoberta. Participaram do estudo 75 pacientes com idade entre 55 e 85 anos, peso menor que $100 \mathrm{~kg}$, estado físico ASA I, II ou III submetidos à cirurgia de catarata sob bloqueio peribulbar e em regime ambulatorial. Consideraram-se elegíveis todos os pacientes que se apresentavam clinicamente controlados em relação as suas doenças de base, e que, em algum momento do peri-operatório, apresentaram pressão arterial sistólica (PAS) maior que $170 \mathrm{mmHg}$ ou pressão arterial diastólica (PAD) maior que $110 \mathrm{mmHg}$.

Foram verificados e anotados os dados demográficos, doenças associadas, medicações em uso, tempo e condições da alta anestésica.

Distribuíram-se os pacientes de forma aleatória em 3 grupos de 25 pacientes, por meio de sorteio simples e encoberto. Os pacientes do Grupo $\mathrm{C} 2$ receberam $2 \mu \mathrm{g} . \mathrm{kg}^{-1}$ de clonidina, por via venosa, de uma solução contendo $20 \mu \mathrm{g} \cdot \mathrm{ml}^{-1}$ e cinco gotas de cloreto de sódio a 0,9\% por via sublingual (SL). Os pacientes do Grupo C3 receberam $3 \mu \mathrm{g} . \mathrm{kg}^{-1}$ de clonidina, por via venosa, de uma solução contendo $30 \mu \mathrm{g} \cdot \mathrm{ml}^{-1}$ e cinco gotas de cloreto de sódio a $0,9 \%$ por via SL. Os pacientes do Grupo A receberam 5 gotas de nifedipina por via SL e $1 \mathrm{ml} .10$ $\mathrm{kg}^{-1}$ de solução de cloreto de sódio por via venosa.

As pressões arteriais sistólica, média, diastólica, freqüência respiratória e freqüência cardíaca foram verificadas antes da administração do tratamento, a cada 2 minutos depois do tratamento (até 20 minutos), antes do bloqueio anestésico, 2 minutos após o bloqueio e a cada 15 minutos até o final do procedimento cirúrgico.

A anestesia ocular foi realizada com técnica peribulbar com dupla punção, a primeira infero-lateral e a segunda supero-medial. Foram injetados de 7 a $10 \mathrm{ml}$ de solução anestésica contendo bupivacaína a $0,5 \%$, lidocaína a $2 \%$ com adrenalina 1:200.000 e hialuronidase $50 \mathrm{U} \cdot \mathrm{ml}^{-1}$.

Considerou-se como sucesso no tratamento das crises hipertensivas o decréscimo da PAS para valor inferior a 160 $\mathrm{mmHg}$ e hipotensão arterial como PAS menor que 110 $\mathrm{mmHg}$. Nos pacientes que permaneceram hipertensos após 20 minutos do tratamento, administrou-se $25 \mathrm{mg}$ de captopril por via sublingual. Os valores da FC foram considerados normais na faixa entre 50 a 100 bpm, considerando-se bradicardia a FC menor que 50 bpm e taquicardia FC maior que 100 $\mathrm{bpm}$. Anotou-se a presença de arritmia cardíaca, queixa de boca seca, sonolência e outros eventos adversos. A utiliza- ção de outras medicações e tratamentos específicos também foram anotados.

As variáveis expressas em freqüência relativa foram analisadas estatisticamente pelos testes do Qui-quadrado ou de Fisher. Dados expressos em médias foram analisados pelos testes de Mann-Whitney ou pareado de Friedman para análises pareadas intragrupo e análise de variância para variáveis repetidas. O tempo de início da ação das drogas foi determinado verificando-se o primeiro momento em que ocorreu diferença estatística em comparação intragrupo com as medidas antes do tratamento.

\section{RESULTADOS}

Os dados demográficos dos dois grupos estão representados na tabela I. Não houve diferenças significativas entre os dois grupos. A prevalência de doenças associadas foi semeIhante nos três grupos e as medicações em uso estão expressas nas tabelas II e III.

Tabela I - Dados Demográficos

\begin{tabular}{lccc}
\hline & Grupo A & Grupo C2 & Grupo C3 \\
\hline Idade $(\mathrm{anos}){ }^{*}$ & $72,6 \pm 7,9$ & $73,8 \pm 11,5$ & $71,2 \pm 7,2$ \\
Peso $(\mathrm{kg})^{*}$ & $63,7 \pm 11,5$ & $70,1 \pm 10.6$ & $71,2 \pm 11,1$ \\
Sexo & & & \\
$\quad$ Masculino & 11 & 12 & 10 \\
$\quad$ Feminino & 14 & 13 & 15 \\
\hline
\end{tabular}

* Valores expressos pela Média \pm DP

Não houve diferença estatística entre os grupos nas variáveis analisadas

Tabela II - Freqüência de Doenças Associadas. Dados Expressos em Incidência e Freqüência Relativa Intragrupo (Porcentagem)

\begin{tabular}{lccc}
\hline & Grupo A & Grupo C2 & Grupo C3 \\
\hline HAS & $17(68 \%)$ & $10(40 \%)$ & $16(64 \%)$ \\
DM & $3(12 \%)$ & $7(28 \%)$ & $3(12 \%)$ \\
DPOC & $1(4 \%)$ & 0 & $1(4 \%)$ \\
ICC & 0 & $2(8 \%)$ & $1(4 \%)$ \\
Disritmia & $1(4 \%)$ & $2(8 \%)$ & $1(4 \%)$ \\
Ansiedade & $3(12 \%)$ & $1(4 \%)$ & $4(16 \%)$ \\
Angina & 0 & $2(8 \%)$ & $1(4 \%)$ \\
\hline
\end{tabular}

As medidas de PAS, PAD e FC antes do tratamento foram semelhantes nos três grupos (tabela IV). Ocorreram diminuições da PAS e PAD $(p<0,001)$ nos três grupos e, em relação a FC, ocorreu diminuição somente nos pacientes tratados com clonidina. Nos grupos $\mathrm{C} 2$ e $\mathrm{C} 3$ as diminuições das PAS e PAD ocorreram precocemente, apresentando significância estatística $(p<0,001)$ após 4 minutos da administração da droga. A tendência de diminuição da PAS se manteve até 12 minutos no grupo C3 e 10 minutos no grupo $\mathrm{C} 2$, verificada pela diferença estatística entre intervalos de tempos subseqüentes. No grupo A, ocorreu diminuição da PAS aos 8 minutos e, a partir de 16 minutos, estabilização dos valores da 
Tabela III - Principais Medicações em Uso. Dados Expressos em Número de Ocorrências e Freqüência Relativa Intragrupo (Porcentagem)

\begin{tabular}{lccc}
\hline & Grupo A & Grupo C2 & Grupo C3 \\
\hline$\beta$-bloqueadores & $4(16 \%)$ & $2(8 \%)$ & $3(12 \%)$ \\
Inibidor de ECA & $2(8 \%)$ & $1(4 \%)$ & $5(20 \%)$ \\
Bloqueadores de canal Ca ${ }^{++}$ & $5(20 \%)$ & $5(20 \%)$ & $7(28 \%)$ \\
Metildopa & $5(20 \%)$ & $2(8 \%)$ & $3(12 \%)$ \\
Diuréticos & $5(20 \%)$ & $5(20 \%)$ & $2(8 \%)$ \\
Digitálicos & 0 & $2(8 \%)$ & $1(4 \%)$ \\
Aspirina & $1(4 \%)$ & $2(8 \%)$ & 0 \\
Hipoglicemiantes orais & $2(8 \%)$ & $4(16 \%)$ & $2(8 \%)$ \\
Insulina & $1(4 \%)$ & $3(12 \%)$ & 0 \\
Antidepressivos & $2(8 \%)$ & $1(4 \%)$ & $1(4 \%)$ \\
\hline
\end{tabular}

Tabela IV - Pressão Arterial Sistólica, Pressão Arterial Diastólica e Freqüência Cardíaca antes do Tratamento (Média $\pm \mathrm{DP}$ )

\begin{tabular}{lccc}
\hline & Grupo A & Grupo C2 & Grupo C3 \\
\hline PAS (mmHg) & $189,3 \pm 14,1$ & $184,5 \pm 14,7$ & $181,2 \pm 8,5$ \\
PAD (mmHg) & $102,5 \pm 10,3$ & $104,2 \pm 11,0$ & $102,3 \pm 8,8$ \\
FC (bpm) & $76,7 \pm 10,5$ & $76,8 \pm 11,4$ & $74,7 \pm 12,6$ \\
\hline
\end{tabular}

PAS (Figura 1). Na comparação dos valores de PAS entre os grupos, houve diferenças entre os grupos $\mathrm{C} 2$ e $\mathrm{C} 3$ e o grupo $\mathrm{A}$ a partir de 2 minutos do tratamento (Figura 1). Resultados semelhantes ocorreram para a PAD (Figura 2).

No grupo $\mathrm{C} 2$ ocorreu diminuição da FC aos 14 minutos, permanecendo estável a partir deste momento. No grupo C3, a diminuição ocorreu aos 10 minutos. Já no grupo A, não ocorreu alteração da FC (Figura 3).

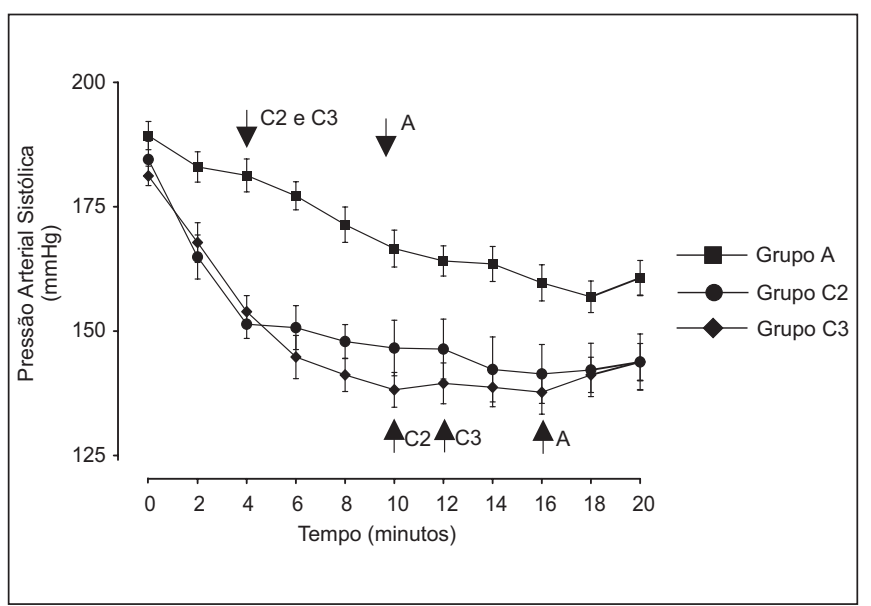

Figura 1 - Valores medidos de PAS em mmHg (Médias \pm DP) em relação ao tempo decorrido do tratamento. Grupo A, que foi tratado com nifedipina e grupos $\mathrm{C} 2$ e C3 que foram tratados com clonidina 2 e $3 \mu \mathrm{g} . \mathrm{kg}^{-1}$. O símbolo $\downarrow$ mostra a partir de qual tempo ocorreu diferença estatística intragrupo em relação as medidas do tempo zero $(p<0,05)$. O símbolo $\uparrow$ mostra a partir de qual tempo ocorreu estabilização dos valores pressóricos. Na comparação entre os três grupos houve diferença estatística a partir de 2 minutos da administração das drogas $(p<0,05)$

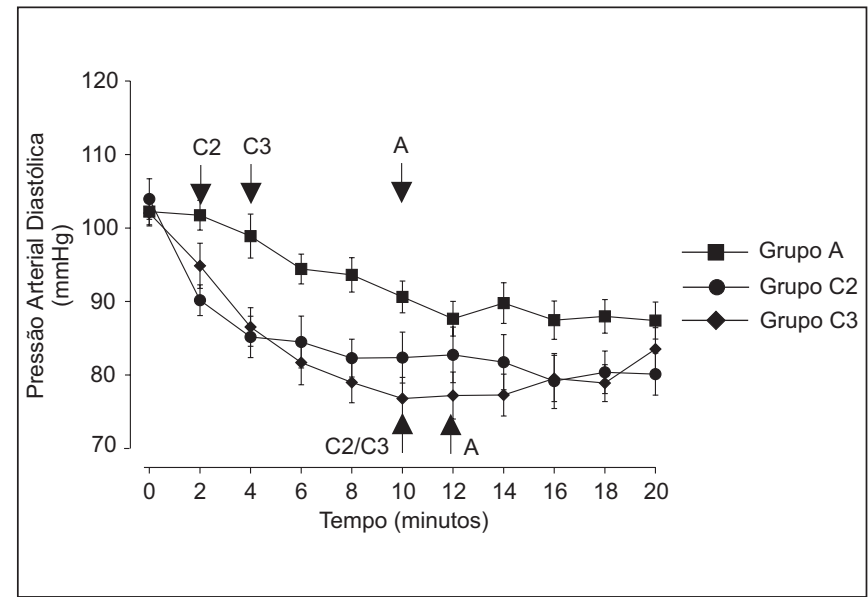

Figura 2 - Valores medidos de PAS em mmHg (Média \pm DP) em relação ao tempo decorrido do tratamento. Grupo A, que foi tratado com nifedipina e grupos $\mathrm{C} 2$ e C3 que foram tratados com clonidina 2 e $3 \mu \mathrm{g} . \mathrm{kg}^{-}$. O símbolo $\downarrow$ mostra a partir de qual tempo ocorreu diferença estatística intragrupo em relação as medidas do tempo zero $(p<0,05)$. O símbolo $\uparrow$ mostra a partir de qual tempo ocorreu estabilização dos valores pressóricos. Na comparaccão entre os três grupos houve diferença estatística a partir de 2 minutos da administração das drogas $(p<0,05)$

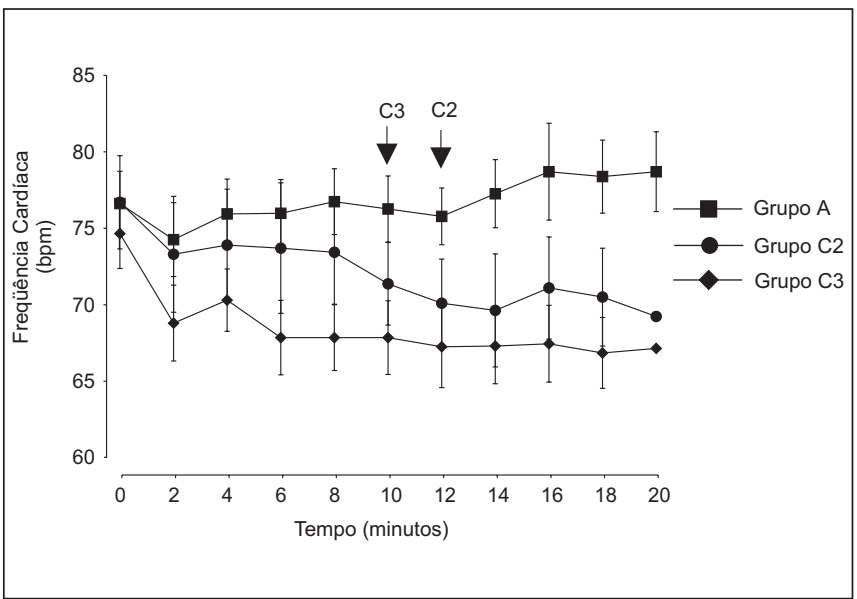

Figura 3 - Valores medidos de FC em ppm (Média \pm DP) em relação ao tempo decorrido do tratamento. Grupo A, que foi tratado com nifedipina e grupos $\mathrm{C} 2$ e C3 que foram tratados com clonidina 2 e $3 \mu \mathrm{g}^{\mathrm{kg}}{ }^{-1}$. Houve diferença estatística entre os grupos C2 e C3 e grupo A $(p<0,05)$. O símbolo $\downarrow$ mostra a partir de qual tempo ocorreu diferença estatística em relação as medidas do tempo zero (análise intragrupo, $p<0,05$ ). No grupo $A$ não houve alteração da FC

A clonidina foi mais efetiva no controle da crise hipertensiva que a nifedipina, demonstrada pela porcentagem de pacientes com PA controlada (PAS $<160 \mathrm{mmHg}$ ), respectivamente, $32 \%, 64 \%$ e $72 \%$ para os grupos $A, C 2$ e C3 $(p<0,05)$. A tabela $\mathrm{V}$ mostra a ocorrência de eventos adversos nos três grupos. O grupo $\mathrm{C} 3$ apresentou mais eventos adversos que os grupos C2 e A. Dentre os eventos adversos mais freqüentes 
destacam-se a sonolência e a queixa de boca seca, sendo que em três pacientes houve a necessidade de se umedecer a orofaringe no per-operatório. Destaca-se também o fato de um paciente do grupo C3 ter apresentado hipotensão arterial intensa (PAS de $80 \mathrm{mmHg}$ ), necessitando de administração de líquidos e de vasopressor.

Tabela V - Incidência de Eventos Adversos

\begin{tabular}{lccc}
\hline & Grupo A & Grupo C2 & Grupo C3 \\
\hline Hipotensão arterial & 1 & 0 & 1 \\
Disritmia & 3 & 2 & 3 \\
Bradicardia & 0 & 0 & 1 \\
Agitação & 3 & 0 & 0 \\
Sonolência & 0 & 3 & $10^{*}$ \\
Angina & 1 & 1 & 0 \\
Vômito & 1 & 0 & 0 \\
Boca seca & 1 & 5 & $7^{*}$ \\
\hline Total & $10 / 25$ & $11 / 25$ & $22 / 25$ \\
\hline
\end{tabular}

* Diferença estatística $(p<0,05)$

\section{DISCUSSÃO}

Nossos resultados estão de acordo com estudos que demonstram que os pacientes portadores de catarata, em geral, são pacientes acima dos 55 anos $^{4}$, proporcionando uma alta incidência de doenças associadas aos sistemas cardiocirculatório e respiratório ${ }^{5}$. Crises hipertensivas em pacientes submetidos à facectomia podem apresentar várias causas, dentre elas, a interrupção das medicações anti-hipertensivas habituais, medo da anestesia, insegurança em relação ao resultado cirúrgico e ao uso de soluções de fenilefrina a $10 \%$ para se obter midríase ${ }^{6}$.

A clonidina é um $\alpha_{2}$-agonista de ação central que apresenta efeito hipotensor sistêmico por diminuir o tônus do sistema nervoso simpático, promovendo estabilidade hemodinâmica ${ }^{7}$. Além de seu efeito hipotensor, a clonidina diminui a pressão intra-ocular ${ }^{8}$, promove sedação, ansiólise, diminui a produção salivar e a concentração de catecolaminas circulantes ${ }^{9}$.

Em estudo anterior, verificamos que a clonidina foi eficaz em controlar crises hipertensivas no peri-operatório de cirurgia de catarata em $95 \%$ dos casos ${ }^{1}$. No estudo atual, encontramos menor eficácia da clonidina do que a encontrada anteriormente, independentemente da dose administrada. Esta diferença deve-se, principalmente, a dois motivos: no primeiro estudo foi considerado tratamento com sucesso quando a PAS diminuía para valores inferiores a $170 \mathrm{mmHg}$, enquanto neste estudo o valor limite foi menor $(160 \mathrm{mmHg})$; em segundo, devido à menor acurácia dos valores obtidos retrospectivamente no primeiro estudo. Somente $32 \%$ dos pacientes tratados com nifedipina tiveram a pressão arterial controlada, enquanto nos grupos C2 e C3 o índice foi de $64 \%$ e $72 \%$, respectivamente.

A clonidina, independentemente da dose administrada, apresentou início de ação e efeito clínico máximo mais rápido que a nifedipina, permitindo, na prática, controle clínico mais rápido e apurado. Após a administração de clonidina, por via venosa, podem ocorrer aumento da pressão arterial e diminuição do débito cardíaco nos primeiros dois minutos ${ }^{10}$. Em nosso estudo não verificamos estas variações devido ao intervalo de dois minutos entre as medidas hemodinâmicas de forma não invasiva. A diminuição precoce dos valores pressóricos e da freqüência cardíaca nos grupos que receberam clonidina estão de acordo com estudo da farmacocinética da clonidina por via venosa, que demonstrou que o tempo de distribuição da droga é em torno de 10 minutos ${ }^{11}$.

Estes dados demonstraram a maior eficácia da clonidina em relação à nifedipina no controle de crises hipertensivas. Entretanto, a dose de $3 \mu \mathrm{g} . \mathrm{kg}^{-1}$ de clonidina esteve associada à alta incidência de sonolência e diminuição da produção salivar. Asonolência obtida em $40 \%$ dos pacientes que receberam $3 \mu \mathrm{g} . \mathrm{kg}^{-1}$ também pode dificultar o ato cirúrgico em cirurgia oftalmológica sob anestesia local, devido à possibilidade de obstruir parcialmente as vias aéreas superiores e, conseqüentemente, levar à movimentação da cabeça durante o esforço inspiratório. Já a diminuição da produção salivar pode provocar desconforto e irritação faríngea e, conseqüentemente, em pelo menos três pacientes do grupo C3 foi necessário umedecer a orofaringe com pequenas quantidades de água.

A associação de clonidina $\operatorname{com} \beta$-bloqueadores pode produzir bradicardia refratária à atropina ${ }^{12}$. Neste estudo, como em dois estudos anteriores, a incidência de bradicardia foi pequena, sendo que no único caso presente não houve a necessidade de tratamento específico. Já a nifedipina, por ser um bloqueador de canal de cálcio de ação periférica, produz vasodilatação com conseqüente taquicardia reflexa ${ }^{1}$. Não ocorreu taquicardia em nosso estudo, provavelmente, por ter sido realizado com população idosa em que o tônus parassimpático prevalece.

Conseqüente à alta incidência de doenças associadas nesta população (Tabela II), a utilização de medicações sistêmicas também é freqüente (Tabela III). A baixa incidência de complicações grave demonstra que é segura a utilização tanto da clonidina quanto da nifedipina na vigência de doenças sistêmicas controladas e de medicações para o seu controle.

Nas condições deste estudo, a clonidina apresentou-se mais eficaz em relação a nifedipina no controle da pressão arterial em pacientes em crise hipertensiva no peri-operatório de cirurgia de catarata. A dose inicial de $2 \mu \mathrm{g} . \mathrm{kg}^{-1}$ de clonidina parece ser mais segura e adequada que a dose de 3 $\mu \mathrm{g} . \mathrm{kg}^{-1} \mathrm{e}$, havendo necessidade, após 15 minutos pode-se aumentar a dose administrada. 


\section{Comparison between Sublingual Nifedipine and Intravenous Clonidine to Control Perioperative Arterial Hypertension in Cataract Procedures}

Renato Mestriner Stocche, TSA, M.D., Luis Vicente Garcia, TSA, M.D., Jyrson Guilherme Klamt, TSA, M.D., Alexandre Pachione, TSA, M.D., Huang H Yu, M.D., Waleska A Oliveira M.D.

\section{INTRODUCTION}

A previous retrospective study has shown the efficacy of intravenous clonidine in controlling perioperative arterial hypertension during cataract extractions ${ }^{1}$. This study has adopted the fixed dose of $150 \mu \mathrm{g}$, regardless of patients' weight. Another placebo-controlled study has observed that premedication with $150 \mu \mathrm{g}$ oral clonidine would prevent arterial hypertension without adverse effects in patients submitted to cataract extractions ${ }^{2}$.

Sublingual nifedipine has been widely used to treat arterial hypertension related or not to surgical procedures ${ }^{3}$. However, we have not found in the literature comparative studies between nifedipine and clonidine to control perioperative pressure.

This study aimed at comparing the efficacy of sublingual nifedipine and 2 intravenous clonidine doses to treat perioperative arterial hypertension during cataract surgery.

\section{METHODS}

After Hospital das Clinicas, Ribeirão Preto Ethical Committee approval and their informed and free consent, participated in this randomized double-blind study 75 patients aged 55 to 85 years, weighting less than $100 \mathrm{~kg}$, physical status ASAI, II or III, submitted to outpatient cataract surgery under peribulbar block. All patients with their underlying disease clinically controlled or who, in a certain perioperative moment, presented systolic blood pressure (SBP) higher than 170 $\mathrm{mmHg}$ or diastolic blood pressure (DBP) higher than 110 $\mathrm{mmHg}$ were eligible for the study.

Demographics data, associated diseases, medications, anesthesia duration and discharge conditions were observed and recorded.

Patients were randomly distributed in three groups of 25 patients by simple and blind drawing. Group $\mathrm{C} 2$ patients received $2 \mu \mathrm{g} \cdot \mathrm{kg}^{-1}$ intravenous clonidine from a solution containing 20 $\mu \mathrm{g} \cdot \mathrm{ml}^{-1}$, and 5 drops of sublingual $0.9 \%$ sodium chloride (SC). Group C3 patients received $3 \mu \mathrm{g} . \mathrm{kg}^{-1}$ intravenous clonidine from a solution containing $30 \mu \mathrm{g} \cdot \mathrm{ml}^{-1}$, and 5 drops of sublingual $0.9 \%$ sodium chloride. Group A patients received 5

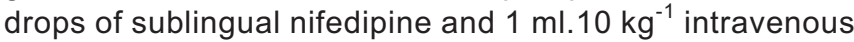
sodium chloride.

Systolic, mean and diastolic pressures, respiratory and heart rates were recorded before treatment and then at 2 minutes intervals (up to 20 minutes) before anesthetic induction, 2 mi- nutes after blockade and at 15 minutes intervals until surgery completion.

Ocular anesthesia was induced by the peribulbar double-puncture technique, being the first infero-lateral and the second supero-medial. Blockade was induced with 7 to $10 \mathrm{ml}$ anesthetic solution with $0.5 \%$ bupivacaine, $2 \%$ lidocaine with epinephrine $1: 200,000$ and $50 \mathrm{U} \cdot \mathrm{ml}^{-1}$ hyaluronidase. Arterial hypertension control was considered a success when SBP < $160 \mathrm{mmHg}$ and arterial hypotension was defined as SBP < $110 \mathrm{mmHg}$. For patients still hypertensive 20 minutes after treatment, $25 \mathrm{mg}$ sublingual captopril were administered. HR was considered normal when in the range of 50 to $100 \mathrm{bpm}$, considering bradycardia when $\mathrm{HR}<50 \mathrm{bpm}$ and tachycardia when HR > 100 bpm. Adverse effects, such as arrhythmia's, dry mouth and somnolence were also recorded, in addition to other medications and specific treatments. Variables expressed in relative frequency were statistically analyzed by Chi-square or Fisher tests. Data expressed in means were analyzed by Mann-Whitney or Friedman paired test for intragroup analysis, and repetitive measures were analyzed by analysis of variance. Onset of drugs was determined at the first moment when there has been a statistical intra-group difference as compared to measurements before treatment.

\section{RESULTS}

Demographics data are shown in table I. There have been no significant differences among groups. The prevalence of associated diseases was similar for all groups and medications used are expressed in tables II and III.

Table I - Demographics Data

\begin{tabular}{lccc}
\hline & Group A & Group C2 & Group C3 \\
\hline Age (years) ${ }^{*}$ & $72.6 \pm 7.9$ & $73.8 \pm 11.5$ & $71.2 \pm 7.2$ \\
Weight $(\mathrm{kg})^{*}$ & $63.7 \pm 11.5$ & $70.1 \pm 10.6$ & $71.2 \pm 11.1$ \\
Gender & & & \\
$\quad$ Male & 11 & 12 & 10 \\
$\quad$ Female & 14 & 13 & 15 \\
\hline
\end{tabular}

*Values expressed in Mean \pm SD

Table II - Frequency of Associated Diseases. Data on Incidence and Relative Intra-Group Frequency (Percentage)

\begin{tabular}{lccc}
\hline & Group A & Group C2 & Group C3 \\
\hline SAH & $17(68 \%)$ & $10(40 \%)$ & $16(64 \%)$ \\
DM & $3(12 \%)$ & $7(28 \%)$ & $3(12 \%)$ \\
CPOD & $1(4 \%)$ & 0 & $1(4 \%)$ \\
$\mathrm{CCl}$ & 0 & $2(8 \%)$ & $1(4 \%)$ \\
Arrhythmia & $1(4 \%)$ & $2(8 \%)$ & $1(4 \%)$ \\
Anxiety & $3(12 \%)$ & $1(4 \%)$ & $4(16 \%)$ \\
Angina & 0 & $2(8 \%)$ & $1(4 \%)$ \\
\hline
\end{tabular}


Table III - Major Medications Used. Data in Number of Occurrences and Relative Intra-Group Frequency (Percentage)

\begin{tabular}{lccc}
\hline & Group A & Group C2 & Group C3 \\
\hline$\beta$-blockers & $4(16 \%)$ & $2(8 \%)$ & $3(12 \%)$ \\
ECA inhibitor & $2(8 \%)$ & $1(4 \%)$ & $5(20 \%)$ \\
Ca $^{++}$channel blockers & $5(20 \%)$ & $5(20 \%)$ & $7(28 \%)$ \\
Methyldopa & $5(20 \%)$ & $2(8 \%)$ & $3(12 \%)$ \\
Diuretics & $5(20 \%)$ & $5(20 \%)$ & $2(8 \%)$ \\
Digitalis & 0 & $2(8 \%)$ & $1(4 \%)$ \\
Aspirin & $1(4 \%)$ & $2(8 \%)$ & 0 \\
Oral hypoglycemics & $2(8 \%)$ & $4(16 \%)$ & $2(8 \%)$ \\
Insulin & $1(4 \%)$ & $3(12 \%)$ & 0 \\
Antidepressants & $2(8 \%)$ & $1(4 \%)$ & $1(4 \%)$ \\
\hline
\end{tabular}

SBP, DBP and HR before treatment were similar among groups (Table IV). All groups presented SBP and DBP decreases $(p<0.001)$ and only clonidine-treated patients had HR decrease. Groups C2 and C3 had early SBP and DBP decreases with statistical significance 4 minutes after drug administration $(p<0.001)$. The trend for SBP decrease was maintained up to 12 minutes in group C3 and up to 10 minutes in group C2, confirmed by statistical differences between subsequent time intervals. Group A presented SBP decrease after 8 minutes and SBP values were stabilized as from $16 \mathrm{mi}-$ nutes (Figure 1). In comparing SBP among groups, there have been differences between groups $\mathrm{C} 2$ and $\mathrm{C} 3$ and group Aas from 2 minutes after treatment (Figure 1). Similar results were obtained for DBP (Figure 2).

$\mathrm{HR}$ decreased in group $\mathrm{C} 2$ at 14 minutes and remained stable afterwards. HR decreased in group $\mathrm{C} 3$ at 10 minutes. There have been no HR changes in group A (Figure 3 ).

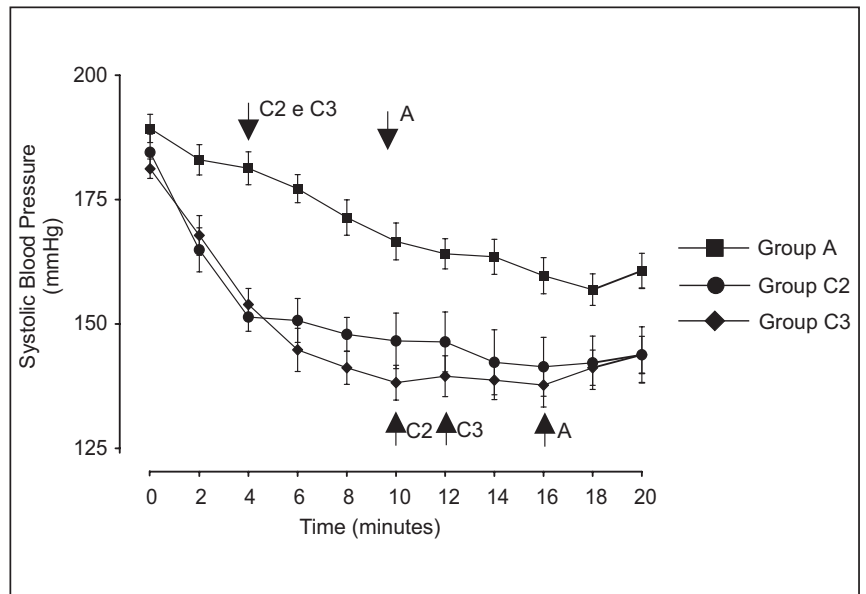

Figure 1 - SBP values measured in $\mathrm{mmHg}$ (Mean $\pm \mathrm{SD}$ ) as compared to time after treatment. Group A, treated with nifedipine, and groups $\mathrm{C} 2$ and $\mathrm{C} 3$, treated with 2 e $3 \mu \mathrm{g} . \mathrm{kg}^{-1}$ clonidine. The symbol $\downarrow$ shows the time when there has been the first intra-group statistical difference as compared to time zero measurements $(p<0.05)$. The symbol $\uparrow$ shows the time when there has been the first pressure stabilization. In comparing the three groups, there has been a statistical difference 2 minutes after drug administration $(p<0.05)$

Revista Brasileira de Anestesiologia

Vol. 52, No 4, Julho - Agosto, 2002
Table IV - Systolic Blood Pressure (SBP), Diastolic Blood Pressure $(\mathrm{DBP})$ and Heart Rate $(\mathrm{HR})$ before Treatment (Mean $\pm \mathrm{SD}$ )

\begin{tabular}{lccc}
\hline & Group A & Group C2 & Group C3 \\
\hline SBP $(\mathrm{mmHg})$ & $189.3 \pm 14.1$ & $184.5 \pm 14.7$ & $181.2 \pm 8.5$ \\
DBP $(\mathrm{mmHg})$ & $102.5 \pm 10.3$ & $104.2 \pm 11.0$ & $102.3 \pm 8.8$ \\
HR $(\mathrm{bpm})$ & $76.7 \pm 10.5$ & $76.8 \pm 11.4$ & $74.7 \pm 12.6$ \\
\hline
\end{tabular}

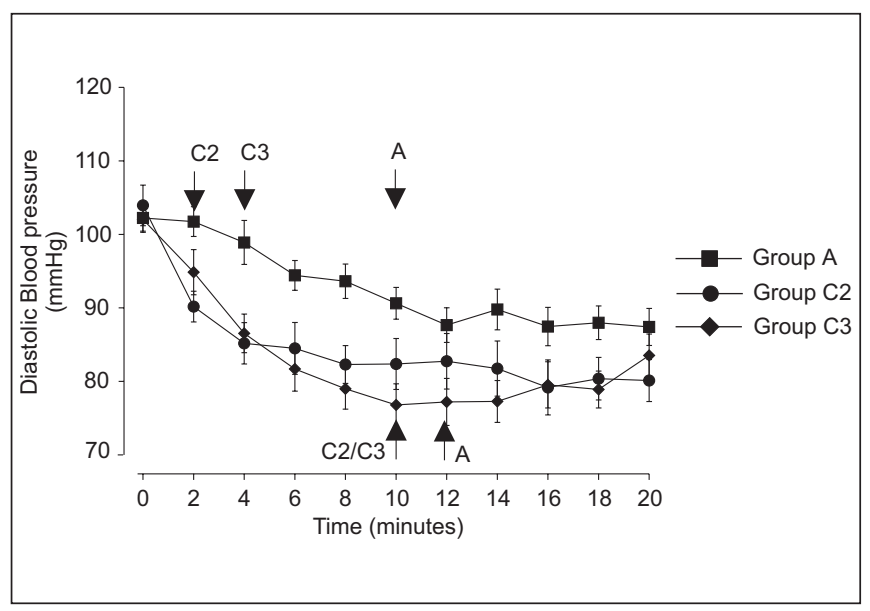

Figure 2 - DBP values measured in $\mathrm{mmHg}$ (Mean $\pm \mathrm{SD}$ ) as compared to time after treatment. Group A, treated with nifedipine, and groups $\mathrm{C} 2$ and $\mathrm{C} 3$, treated with 2 e $3 \mu \mathrm{g} \cdot \mathrm{kg}^{-1}$ clonidine. The symbol $\downarrow$ shows the time when there has been the first intra-group statistical difference as compared to time zero measurements $(p<0.05)$. The symbol $\uparrow$ shows the time when there has been the first pressure stabilization. In comparing the three groups, there has been a statistical difference 2 minutes after drug administration $(p<0.05)$

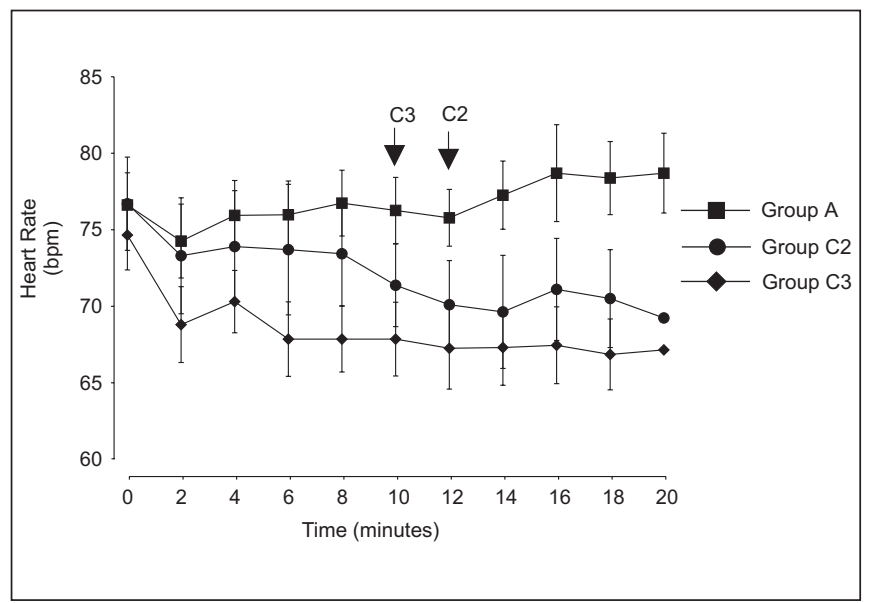

Figure 3 - HR values measured in ppm (Mean $\pm \mathrm{SD}$ ) as compared to time after treatment. Group A, treated with nifedipine, and groups C2 and C3, treated with 2 e $3 \mu \mathrm{g} \mathrm{kg}^{-1}$ clonidine. There has been a statistical difference between groups C2 and C3 and group $A(p<0.05)$. The symbol $\downarrow$ shows the time when there has been the first intra-group statistical difference as compared to time zero measurements (intra-group analysis, $p<$ 0.05). Group A has not shown HR changes 
Clonidine was more effective in controlling arterial hypertension as compared to nifedipine, as shown by the percentage of patients with controlled BP (SBP $<160 \mathrm{mmHg}$ ), respectively $32 \%, 64 \%$ and $72 \%$ for groups $A, C 2$ and $C 3(p<0.05)$. Table $\mathrm{V}$ shows the incidence of adverse events in all groups. Group C3 had more adverse events as compared to groups $\mathrm{C} 2$ and $\mathrm{A}$. Among most frequent adverse events, somnolence and dry mouth are to be highlighted and three patients needed perioperative oropharyngeal moistening. It is also to be stressed that one of group C3 patient had a severe arterial hypotension (SBP $=80 \mathrm{mmHg}$ ) and had to receive fluids and vasopressants.

Table V - Incidence of Adverse Effects

\begin{tabular}{lccc}
\hline & Group A & Group C2 & Group C3 \\
\hline Arterial hypotension & 1 & 0 & 1 \\
Arrhythmia & 3 & 2 & 3 \\
Bradycardia & 0 & 0 & 1 \\
Agitation & 3 & 0 & 0 \\
Somnolence & 0 & 3 & $10^{*}$ \\
Angina & 1 & 1 & 0 \\
Vomiting & 1 & 0 & 0 \\
Dry mouth & 1 & 5 & $7^{*}$ \\
\hline Total & $10 / 25$ & $11 / 25$ & $22 / 25$ \\
\hline
\end{tabular}

* Statistical difference $(p<0.05)$

\section{DISCUSSION}

Our results are in line with studies which have shown that cataract patients in general are patients above 55 years of age ${ }^{4}$, with a high incidence of associated cardiocirculatory and respiratory diseases ${ }^{5}$. Arterial hypertension in patients submitted to cataract extraction may have different causes, among them, withdrawal of regular anti-hypertensive medication, fear of anesthesia, uncertainty as to surgical result, and the use of $10 \%$ phenylephrine to obtain midriasis ${ }^{6}$.

Clonidine is a central action $\alpha_{2}$-agonist with systemic hypotensive effect by decreasing sympathetic nervous system tone, thus promoting hemodynamic stability ${ }^{7}$. In addition to its hypotensive effect, clonidine decreases intraocular pressure $^{8}$, promotes sedation and anxiolysis, decreases saliva production and circulating catecholamines concentration ${ }^{9}$.

In a previous study, we have observed that clonidine was $95 \%$ effective in controlling perioperative arterial hypertension during cataract procedures ${ }^{1}$. In this study, we have found a lower efficacy of clonidine as compared to our previous study, regardless of the dose. This difference is mainly due to two reasons: the first study considered the treatment a success when SBP would decrease below $170 \mathrm{mmHg}$, while in this study the limit value was lower $(160 \mathrm{mmHg})$; secondly, the poorer accuracy of values retrospectively obtained in the first study. Only $32 \%$ of patients treated with nifedipine had their pressures controlled, while in groups $\mathrm{C} 2$ and $\mathrm{C} 3$ the rate was $64 \%$ and $72 \%$, respectively.
Clonidine, regardless of the dose, had a faster onset and maximum clinical effect as compared to nifedipine, thus allowing for a faster and more accurate clinical control. After the administration of intravenous clonidine, there may be blood pressure increase and cardiac output decrease in the first 2 minutes ${ }^{10}$. Such changes were not seen in our study due to the 2 minutes interval between non-invasive hemodynamic measurements. The early pressure and heart rate decrease in the clonidine groups is in line with pharmacokinetic studies of intravenous clonidine, which have shown that time for drug spread is around 10 minutes ${ }^{11}$.

Such data have shown a better efficacy of clonidine as compared to nifedipine to control arterial hypertension. However, the $3 \mu \mathrm{g} . \mathrm{kg}^{-1}$ dose was associated to a high incidence of somnolence and dry mouth. Somnolence observed in $40 \%$ of patients receiving $3 \mu \mathrm{g} . \mathrm{kg}^{-1}$ may also impair the surgical procedure in ophthalmic surgeries under local anesthesia due to the possibility of partially obstructing upper airways and, as a consequence, lead to head movement during inspiratory effort. Dry mouth, on the other hand, may cause discomfort and pharyngeal irritation and, as a consequence, at least three patients needed oropharyngeal moistening with small amounts of water.

The association of clonidine and $\beta$-blockers may produce atropine-refractory bradycardia ${ }^{12}$. In our study, as well as in two previous studies, there has been a minor incidence of bradycardia and in the only case there has been no need for specific treatment. Nifedipine, for being a peripheral action calcium channel blocker, causes vasodilatation with a consequent reflex tachycardia ${ }^{1}$. There has been no tachycardia in our study, probably because it was performed with an elderly population where there is a prevalence of parasympathetic tone.

As a consequence of the high incidence of associated diseases in this population (Table II) the use of systemic medication was also frequent (Table III). The low incidence of severe complications has shown that it is safe to use both clonidine and nifedipine in the presence of controlled systemic diseases and medications for their control.

In the conditions of this study, clonidine was more effective as compared to nifedipine in controlling perioperative arterial hypertension during cataract extractions. The initial $2 \mu \mathrm{g} \cdot \mathrm{kg}^{-1}$ clonidine dose seems to be safer and more adequate than the $3 \mu \mathrm{g} \cdot \mathrm{kg}^{-1}$ dose and the dose may always be increased after 15 minutes, if needed.

\section{REFERÊNCIAS - REFERENCES}

01. Stocche RM, Garcia LV, Klamt JG - Clonidina venosa no controle da hipertensão arterial perioperatória em cirurgias de catarata. Estudo retrospectivo. Rev Bras Anestesiol, 2000;50:289-293.

02. Stocche RM, Garcia LV, Klamt JG - Medicação pré-anestésica com clonidina por via oral em cirurgia de catarata. Rev Bras Anestesiol, 2000;50:278-282.

03. Reves JG - The relative hemodynamic effects of $\mathrm{CA}^{++}$entry blockers. Anesthesiology, 1984;61:3. 
04. Sperduto RD, Seigel D - Senile lens and senile macular changes in a populational-based sample. Am J Ophthalmol, 1980;90: 86-91.

05. Drolsum L, Haaskjold E - The influence of age on characteristics of cataract patients. Acta Ophthalmol, 1994;72:622-626.

06. Pascoe PJ, Ilkiw JE, Stiles J et al - Arterial hypertension associated with topical ocular use of phenylephrine in dogs. J Am Vet Med Assoc, 1994;205:1562-1564.

07. Laurito CE, Baughman VL, Becker GL et al - The effectiveness of oral clonidine as a sedative/anxiolytic and as a drug to blunt the hemodynamic responses to laringoscopy. J Clin Anesth, 1991; 3:186-193.

08. Ghignone M, Noe C, Calvillo O et al - Anesthesia for ophthalmic surgery in the elderly: the effects of clonidine on intraocular pressure, perioperative hemodynamics, and anesthetic requirement. Anesthesiology, 1988;68:707-716.

09. Howie MB, Hiestand DC, Jopling MW et al - Effect of oral clonidine pre medication on anesthetic requirement, hormonal response, hemodynamics, and recovery in coronary artery bypass graft surgery patients. J Clin Anesth, 1996;8:263-272.

10. Lawrence CJ, Prinzen FW, de Lange S - Effects of the specific alpha2 adrenergic agonist dexmedetomidine on the systemic and coronary circulation of the anesthetized goat. J Cardiothorac Vasc Anesth, 1992;92:75.

11. Davies DS, Wing LMH, Reid JL et al - Pharmacokinetics and concentration-effect relationships of intravenous and oral clonidine. Clin Pharmacol Ther, 1977;21:593-601.

12. Stühmeier K D; Mainzer B; Cierpka J et al - Small, oral dose of clonidine reduces the Incidence of intraoperative myocardial ischemia in patients having vascular surgery. Anesthesiology, 1996;85:706-712.

\section{RESUMEN}

Stocche RM, Garcia LV, Klamt JG, Pachione A, Yu HH, Oliveira WA - Comparación entre Nifedipina por Vía Sub-Lingual y Clonidina por Vía Venosa en el Control de Hipertensión Arterial Peri-Operatoria en Cirugías de Catarata

Justificativa y Objetivos - Estudio reciente muestra que la clonidina por vía venosa se presenta eficaz y segura en el tratamiento de crisis hipertensivas durante cirugía de catarata. Este estudio visa comparar el uso de nifedipina, droga ampliamente utilizada por vía sub-lingual, y clonidina por vía venosa en el control de la hipertensión en cirugías de catarata.

Método - Setenta e cinco pacientes sometidos a facectomia fueron distribuidos de forma aleatoria y encubierta en: Grupo A, que recibió nifedipina y Grupos C2 y C3, que recibieron, respectivamente, 2 y $3 \mu \mathrm{g} \cdot \mathrm{kg}^{-1}$ de clonidina por vía venosa. Todos los pacientes presentaban hipertensión arterial (PAS > $170 \mathrm{mmHg}$ o PAD > $110 \mathrm{mmHg}$ ). Las PAS, PAD y frecuencia cardíaca ( $F C$ ) fueron medidas y comparadas en los momentos: 0 (antes del tratamiento) y de 2 en 2 minutos hasta el final del procedimiento. Los eventos adversos fueron anotados.

Resultados - Después del tratamiento ocurrieron diminuciones de la PAS y PAD en los 3 grupos $(p<0,001)$. Hubo control de la presión arterial $(<160 \mathrm{mmHg})$ en $32 \%, 64 \%$ y $72 \%$ de los pacientes en los grupos A, C2 y C3, respectivamente $(p<$ $0,05)$. En el grupo C3 ocurrió mayor incidencia de efectos colaterales que en los grupos C2 y A $(p<0,05)$.

Conclusiones - La clonidina por vía venosa es más eficaz que la nifedipina, por vía sub-lingual, en el control de crisis hipertensivas en el peri-operatorio de cirugías de catarata. Sin embargo, la dosis de $3 \mu \mathrm{g} \cdot \mathrm{kg}^{-1}$ puede estar relacionada a efectos colaterales, debiéndose iniciar el tratamiento con 2 $\mu g \cdot \mathrm{kg}^{-1}$. 